1 Institute of Molecular BioSciences, Massey University, Palmerston North, New Zealand

2 Public Health Laboratory, Countess of Chester Health Park, Chester, UK

3 Department of Oral Sciences and Orthodontics, University of Otago, New Zealand

4 Faculty of Allied Health Sciences, Universiti Kebangsaan Malaysia, Malaysia

5 Microbiology Division, Wellington Hospital, Wellington, New Zealand

6 Microbiology Department Auckland Hospital, Auckland, New Zealand

7 Microbiology Unit, Canterbury Health Laboratories, Christchurch New Zealand

8 Medlab South Ltd, Christchurch, New Zealand

9 Corporacion para Investigaciones Biologicas, Medellin, Colombia

10 Department of Pathology, CWM Hospital, Suva, Fiji

\section{Evidence for a general-purpose genotype in Candida albicans, highly prevalent in multiple geographical regions, patient types and types of infection}

Jan Schmid, ${ }^{1}$ Scott Herd, ${ }^{1}$ Paul R. Hunter, ${ }^{2}$ Richard D. Cannon, ${ }^{3}$ M. Salleh M. Yasin, ${ }^{4}$ Shamin Samad, ${ }^{4}$ Mary Carr, ${ }^{5}$ Dinah Parr, ${ }^{6}$ Wendy McKinney, ${ }^{6}$ Mona Schousboe, ${ }^{7}$ Ben Harris, ${ }^{7}+$ Rosemary Ikram, ${ }^{8}$ Mike Harris, ${ }^{8}$ Angela Restrepo, ${ }^{9}$ Guillermo Hoyos ${ }^{9}$

and Kumar P. Singh ${ }^{10} \neq$

Author for correspondence: Jan Schmid. Tel: +64 6350 4018. Fax: +64 63505637. e-mail: J.Schmid@massey.ac.nz

Epidemiological studies, using the probe Ca3, have shown that in a given patient population a single cluster of genetically related Candida albicans isolates usually predominates. The authors have investigated whether these local clusters are part of a single group, geographically widespread and highly prevalent as an aetiological agent of various types of candidiasis. An unrooted neighbour-joining tree of $\mathbf{2 6 6}$ infection-causing C. albicans isolates (each from a different individual) from 12 geographical regions in 6 countries was created, based on genetic distances generated by $\mathrm{Ca} 3$ fingerprinting. Thirtyseven per cent of all isolates formed a single genetically homogeneous cluster (cluster A). The remainder of isolates were genetically diverse. Using the maximum branch length within cluster $A$ as a cut-off, they could be divided into 37 groups, whose prevalence ranged between $0.3 \%$ and $9 \%$. Strains from cluster A were highly prevalent in all but one geographical region, with a mean prevalence across all regions of $41 \%$. When isolates were separated into groups based on patient characteristics or type of infection, strains from cluster A had a prevalence exceeding $27 \%$ in each group, and their mean prevalence was $43 \%$ across all patient characteristics. These data provide evidence that cluster A constitutes a general-purpose genotype, which is geographically widespread and acts as a predominant aetiological agent of all forms of candidiasis in all categories of patients surveyed.

Keywords: Candida albicans, pathogenesis, evolution, general-purpose genotype, Ca3 fingerprinting

\section{INTRODUCTION}

Ca3 fingerprints have been shown to be reliable markers of genetic relationships between Candida albicans strains, through comparative analysis using other genetic markers - randomly amplified polymorphic DNA

†Present address: Southern Community Laboratories, Christchurch, New Zealand.

¥Present address: Universal Diagnostic Laboratories, Fairfield, Australia.

Abbreviation: UPGMA, unweighted pair group method using arithmetic averages. 
(RAPD), multilocus enzyme electrophoresis (MLEE) and phenotypic markers (Schmid et al., 1995a; Pujol et al., 1997). Using Ca3 fingerprinting, it has been shown (i) that a single cluster of genetically related infectioncausing Candida albicans isolates usually predominates in a given patient population in a given geographical locale (Schmid et al., 1990, 1993, 1995a, b; Hellstein et al., 1993; Pfaller et al., 1998) and (ii) that the genetic distance between isolates from different regions exceeds that between isolates from the same region, indicating regional specificity of C. albicans (Schmid et al., 1993; Pfaller et al., 1998). These observations can be explained in two ways. It is possible that in different geographical regions and/or patient types different groups of strains predominate. Alternatively it is possible that the predominant groups described in these studies are local representatives of one group, geographically widespread and causing infection in a variety of patient types. Such a group would constitute the most important aetiological agent of candidiasis and would warrant focussed attention. If such a group exists, regional specificity would be caused by genetic differences between local subgroups within this group and/or by regional differences in the prevalence of other groups.

To determine if a geographically widespread highly prevalent group of C. albicans strains exists, we have conducted an analysis of the electronically stored fingerprints of isolates from our earlier studies plus isolates from additional regions, covering 266 isolates in 12 geographical regions in 6 countries collected from a variety of patient types.

\section{METHODS}

Sources of isolates, fingerprinting and analysis of trees. The sources of isolates are presented in Table 1. DNA fingerprinting was carried out as described previously (Schmid et al., 1990) by Southern hybridization of EcoRI-digested total Candida DNA with the moderately repetitive sequence Ca3 and by entering recorded molecular masses and intensities of bands into an electronic database. A simple genetic distance between strains was then calculated according to the formula: $D=1-\frac{\sum_{i=1}^{k}\left(a_{i}+b_{i}-\left|a_{i}-b_{i}\right|\right)}{\sum_{i=1}^{k}\left(a_{i}+b_{i}\right)}$

where $a_{i}$ and $b_{i}$ are the intensities of band $i$ in patterns $\mathrm{A}$ and $\mathrm{B}$ respectively, and $k$ is the number of bands. $D$ can range between $1 \cdot 0$ (the two patterns have no bands in common) and $0 \cdot 0$ (the two patterns are identical). An unrooted tree was generated from these distances by neighbour-joining, using PAUP" (version 4.0d63), written by David L. Swofford. Because local tree construction approaches as used in this method do not guarantee an optimal tree (Swofford et al., 1996), the significance of major branches and clusters within the tree was tested using a jackknifing procedure (Lanyon, 1985; Lapointe et al., 1994). For this purpose, trees were generated by Splitstree (version 2.3.2, written by Daniel Huson) for quartets of strains, two randomly chosen from within a putative branch or cluster and two randomly chosen from outside the putative branch or cluster. For each set it was recorded whether the isolation index supported a closer association between the two strains from within the putative branch or cluster than to either of the two strains from without the putative branch or cluster. The frequency with which the strains from putative branches or clusters are associated with each other provides an indication of the significance of the branch or cluster. Branches or clusters were considered as separated from the remaining isolates if this frequency significantly $(z$ test, $P<0.05$ ) exceeded 0.33 , the maximum frequency expected for random association of two isolates in a set of four. This is a conservative estimate of significance, since in some quartets of randomly chosen strains the isolation index will indicate equal separation between all isolates; such quartets will reduce the frequency of random association between isolates below 0.33

\section{RESULTS}

\section{A neighbour-joining tree of 266 C. albicans isolates}

Because our earlier results had raised the possibility that a highly successful group of genetically similar strains might exist which is prevalent in patients in different geographical regions, we generated a tree based on the $\mathrm{Ca} 3$ patterns of 266 infection-causing C. albicans isolates, each from a separate individual, from 12 geographical locations in 6 countries (see Table 1 for sources of isolates). We used neighbour-joining and an unrooted phylogram tree format. This allowed us to observe separation of isolates into branches and clusters without the need for fixed, arbitrarily defined cut-off points, overcoming a major drawback of the UPGMAbased dendrograms used previously by ourselves and others. In order to use neighbour-joining, we converted the similarity $\left(S_{\mathrm{AB}}\right)$ values previously used by us (Schmid et al., 1990) into a simple genetic distance, equivalent to $1-S_{\mathrm{AB}}$ (see Methods). The resulting tree (Fig. 1) showed three major branches, labelled A, B and C in the figure, containing 98, 31 and 137 isolates respectively (i.e. $37 \%$ $12 \%$ and $52 \%$ of all the isolates tested). The separation of each branch from the remaining isolates was verified by jackknifing as described in Methods. Fig. 2 shows Ca3 fingerprints of representatives of the branches.

In one of the branches all isolates were genetically very similar to each other, forming a short-branched cluster (cluster A). The mean distance between any two isolates belonging to the cluster was $0 \cdot 19 \pm 0 \cdot 06$. The cluster appeared subdivided into two halves, containing 37 and 61 isolates respectively (labelled A1 and A2 in Fig. 1) but this division was only weakly supported (see legend to Fig. 1).

In contrast, the branches labelled $\mathrm{B}$ and $\mathrm{C}$ were conglomerates of a large number of genetically different isolates and groups. The mean distance between isolates within branch $B$ was $0.33 \pm 0.09$; within branch $C$ it was $0 \cdot 35 \pm 0 \cdot 09$. The mean distance between any two isolates belonging to either of them was $0 \cdot 37 \pm 0 \cdot 09$. To dem- 
Table 1. Origin and date of isolation of C. albicans strains used in this study

\begin{tabular}{|c|c|c|c|}
\hline Region & $\begin{array}{l}\text { No. of } \\
\text { isolates }\end{array}$ & $\begin{array}{l}\text { Date of } \\
\text { isolation }\end{array}$ & Source* \\
\hline Iowa, USA & 41 & $1984-89$ & $\begin{array}{l}\text { Schmid et al. }(1990,1995 \mathrm{a}) \text {; } \\
\text { Hellstein } \text { et al. }(1993) \text {; this study }\end{array}$ \\
\hline Michigan, USA & 10 & 1990-91 & Schmid et al. (1993) \\
\hline Medellin, Colombia & 11 & 1994-95 & This study \\
\hline Leicester, UK & 9 & $1988-90$ & Schmid et al. (1992) \\
\hline London, UK & 10 & 1986-88 & This study \\
\hline Various, UK† & 10 & $1986-88$ & This study \\
\hline Kuala Lumpur, Malaysia & 12 & 1995 & This study \\
\hline Suva, Fiji & 11 & 1995 & This study \\
\hline Auckland, New Zealand & 51 & 1992-93 & Schmid et al. (1995b) \\
\hline Wellington, New Zealand & 57 & 1992-93 & Schmid et al. (1995b) \\
\hline Christchurch, New Zealand & 26 & 1992-94 & This study \\
\hline Otago, New Zealand & 18 & 1990-91 & This study \\
\hline
\end{tabular}

*Each isolate was obtained from a different patient exhibiting symptoms of candidiasis and/or carrying levels of Candida exceeding those for commensal colonization listed by Odds (1988). See Table 3 for information on patient characteristics and types of infection.

†Three isolates from Bangor, two from Southampton and one each from Oxford, Manchester, Welwyn, Basildon and Cardiff.

onstrate further the diversity within branches B and C we used the genetic distance between the branching point at which cluster A subgroups A1 and A2 unite and the end-point of the longest branch in cluster A (a genetic distance of $0 \cdot 17$ ) for defining cut-off points for groups within branches $\mathrm{B}$ and $\mathrm{C}$, whose members were as similar to each other as the isolates contained within cluster A. Using this approach, branch B could be divided into 11 groups, containing between one and seven isolates (prevalences of $0.3 \%$ to $3 \%$ ). Branch C isolates could be divided into 26 groups, containing between one and twenty-five isolates, i.e. with prevalences ranging from $0.3 \%$ to $9 \%$ [half of the group with $9 \%$ prevalence constituted epidemiologically related isolates from a single hospital (Schmid et al., 1995 b), suggesting a prevalence of this group among epidemiologically unrelated isolates of $4 \cdot 5 \%$ ]. Note that these groups were defined, using an arbitrary threshold, only for the purpose of illustrating the high genetic diversity within branches B and C. Many would not be considered homogeneous groups when submitted to the jackknifing test we used to assess the significance of branches in the tree (see Methods); if such a test were applied they would need to be split further to obtain true (according to jackknifing) groups. As a result, the numbers given above underestimate the 'true' (according to jackknifing) number of groups in branches B and $\mathrm{C}$ containing isolates as closely related as those in cluster A (and consequently overestimate their prevalence). Thus branches $\mathrm{B}$ and $\mathrm{C}$ are split into more than 37 groups of 0.3 to $\leqslant 4.5 \%$ prevalence, as compared to a single group (cluster) with $37 \%$ prevalence, constituting the entire branch A.

\section{Cluster A constitutes a group highly prevalent in different geographical regions}

Cluster A contained a high number of genetically similar isolates suggestive of a genetically homogeneous group, highly prevalent in many geographical regions. When we tested this assumption (Table 2) we found that, although there were apparent differences in prevalence between regions $\left(\chi^{2}\right.$ test, $\left.P<0 \cdot 001\right)$, strains from the cluster were isolated frequently in all but one region. Strains from the cluster constituted more than one-third of all isolates in each region sampled with the exception of three regions in New Zealand. Only in one region (Otago, New Zealand) was their apparent prevalence below $19 \%$. The mean prevalence of cluster A across all regions was $41 \%$. This mean, calculated by giving the same weight to each region, was very similar to the prevalence determined above based on the number of isolates. The same was true for the subgroups within the cluster, A1 and A2 (data not shown), and the distribution of the genetically diverse isolates between branches B and $\mathrm{C}$. This suggests that the tree shown in Fig. 1 gives a fairly accurate impression of the distribution of strains throughout all regions even though the number of isolates included in the tree differed between regions.

Since the isolates from different regions were collected over a period of 11 years, we also tested whether there were any indications that the prevalence of cluster $\mathrm{A}$ had changed over time. Our analysis did not allow us to investigate time-dependent changes within each region sampled, but a regression analysis of the data displayed in Fig. 3 gave no indication of a global trend towards an increase or decrease in prevalence. 


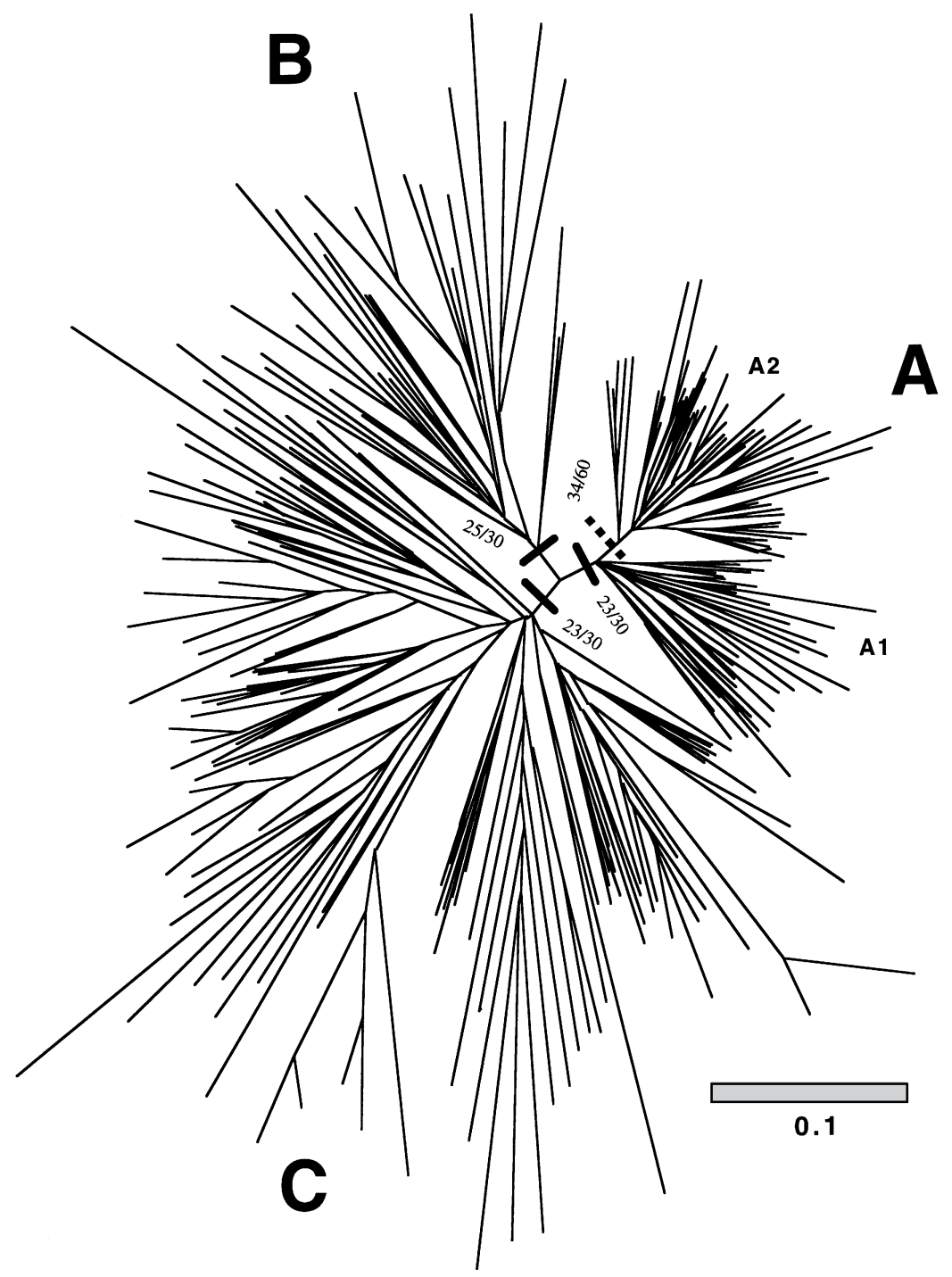

Fig. 1. Neighbour-joining tree of $266 \mathrm{C}$. albicans isolates. The major branches $A$ (equivalent to cluster $\mathrm{A}), \mathrm{B}$ and $\mathrm{C}$ are labelled, as well as the two subgroups of cluster A (A1 and A2). Bars designate the borders of branches and the adjacent numbers the proportions of quartets which supported them when their significance was tested by jackknifing as described in Methods. Initially 30 quartets were analysed. For the division between $\mathrm{A} 1$ and $\mathrm{A} 2,60$ quartets had to be analysed to demonstrate its significance. The bar in the lower part of the figure is equivalent to a genetic distance of $0 \cdot 1$.

\section{Cluster A strains are highly prevalent in a variety of patient types}

The hypothesis that a group of genetically similar strains might exist which is highly prevalent in different regions arose from some of our previous studies which found that in a given region a large percentage of isolates from a target population of patients (such as AIDS patients, patients suffering from vaginitis or bloodstream infections, hospitalized patients) was part of a single cluster of genetically similar strains, with the remainder of isolates falling into numerous smaller groups (Schmid et al., 1992, 1993, 1995a, b). We determined that, with one exception, cluster A strains corresponded to the predominant groups of genetically similar strains defined in these earlier studies, as judged by the position of isolates belonging to these groups in the tree shown in Fig. 1 (data not shown; the exception was a group of related branch $\mathrm{C}$ strains nosocomially transmitted within Wellington hospital - however, among outpatients and recent admissions in Wellington, strains belonging to cluster A again dominated). We also determined that a cluster containing $79 \%$ of all bloodstream isolates in the northeast of the USA in 1995/1996, defined by Pfaller et al. (1998) using Ca3 fingerprinting, coincided with cluster A. Fingerprints of four representative isolates from the cluster defined by Pfaller and coauthors (NYB13, NYB8, NYB1 and Van2) were more similar to those of isolates in cluster A than to other isolates in our sample. The genetic distances between them and their closest relatives in cluster A ranged between $0 \cdot 040$ and $0 \cdot 132$.

These observations suggested that strains from cluster A can be highly prevalent in a wide variety of patient groups and types of infections. However, the studies listed above had defined clusters of Candida strains on the basis of arbitrary cut-offs and covered only a small range of patient characteristics, each assessed either in a single geographical region or in a limited number of adjacent geographical regions. We therefore tested the impact of patient attributes on a broader basis by determining the prevalence of cluster A strains in groups of patients defined by single attributes, including sites of 


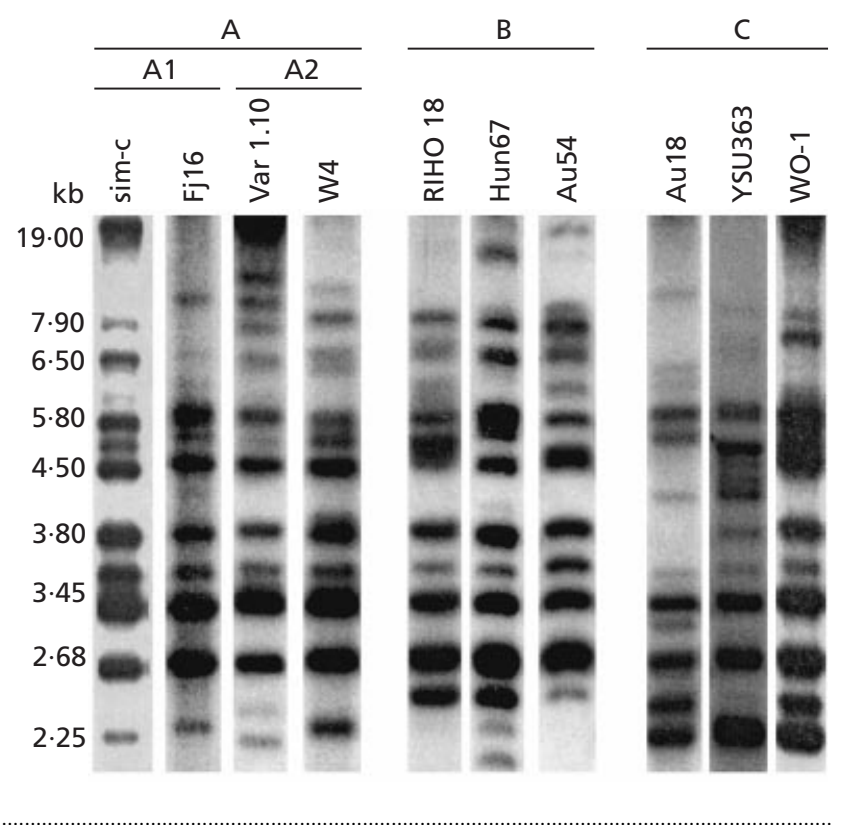

Fig. 2. Examples of DNA fingerprints. Four fingerprints are shown for cluster $A$ (two each for subdivisions $A 1$ and $A 2$, respectively) and three each for isolates belonging to branch $B$ and branch $C$, respectively. Isolate $W 4$ was collected in Wellington, Au18 and Au54 were collected in Auckland, New Zealand; Hun 67 in Bangor, Great Britain; Var1.10 in Michigan, USA; sim-c, RIHO 18 and WO-1 in lowa, USA; Fj16 in Suva, Fiji; and YSU363 in Kuala Lumpur, Malaysia.

infection, across all regions (Table 3). Prevalences of the cluster in the patient groups shown must be interpreted with a degree of caution because they are possibly influenced both by epidemiological connections between isolates within regions and by region-specific differences

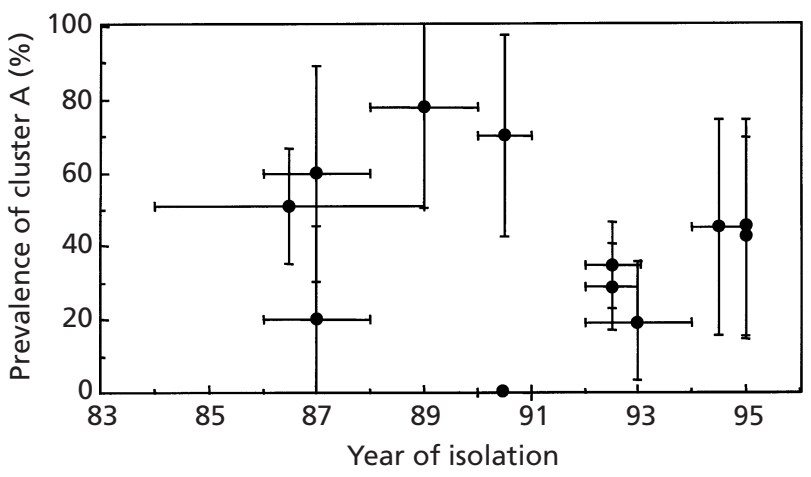

Fig. 3. Prevalence of cluster A strains in samples of isolates collected at different times. Data points refer to the prevalence in each of the sampling regions listed in Table 1 at the median time of collection. Horizontal bars indicate the sampling interval. Vertical bars indicate $95 \%$ confidence limits.

in prevalence (since not all patient characteristics were encountered in all regions). Nevertheless, the data show clearly that cluster A was highly prevalent in all groups. For no characteristic chosen was the prevalence of cluster A strains less than $27 \%$, and it reached $>75 \%$ in one group. We found no statistically significant dependence of the prevalence of cluster A strains on patient age (data not shown).

The mean prevalence of cluster A calculated by giving equal weight to each patient characteristic $(43 \%)$ was almost identical to prevalences giving equal weight to each region or to each strain, mentioned above. The same was true for the distribution of cluster A isolates between subgroups A1 and A2 (data not shown) and for

Table 2. Prevalence of cluster $A$, and of isolates belonging to branches $B$ and $C$, in different geographical regions

\begin{tabular}{|lcccc|}
\hline \multirow{2}{*}{ Region } & \multirow{2}{*}{$\begin{array}{c}\text { No. of } \\
\text { isolates }\end{array}$} & \multicolumn{2}{c|}{ Prevalence in \% $(\mathbf{9 5} \%$ confidence limits) } \\
\cline { 3 - 5 } & & Cluster A & Branch B & Branch C \\
& & & & \\
& & & & \\
Iowa, USA & 41 & $51 \cdot 5(35 \cdot 9-66 \cdot 5)$ & $14 \cdot 6(3 \cdot 8-25 \cdot 5)$ & $34 \cdot 1(19 \cdot 6-48 \cdot 7)$ \\
Michigan, USA & 10 & $70 \cdot 0(41 \cdot 6-98 \cdot 4)$ & $20 \cdot 0(0-44 \cdot 8)$ & $10 \cdot 0(0-28 \cdot 6)$ \\
Leicester, UK & 9 & $77 \cdot 8(50 \cdot 6-100 \cdot 0)$ & $11 \cdot 1(0-31 \cdot 6)$ & $11 \cdot 1(0-31 \cdot 6)$ \\
London, UK & 10 & $60 \cdot 0(29 \cdot 6-90 \cdot 4)$ & $20 \cdot 0(0-44 \cdot 8)$ & $20 \cdot 0(0-44 \cdot 8)$ \\
Various, UK & 10 & $20 \cdot 0(0-44 \cdot 8)$ & $20 \cdot 0(0-44 \cdot 8)$ & $60 \cdot 0(29 \cdot 6-90 \cdot 4)$ \\
Auckland, NZ & 51 & $29 \cdot 4(16 \cdot 9-41 \cdot 9)$ & $13 \cdot 7(4 \cdot 3-23 \cdot 2)$ & $56 \cdot 9(43 \cdot 3-70 \cdot 5)$ \\
Wellington, NZ & 57 & $35 \cdot 1(22 \cdot 7-47 \cdot 5)$ & $12 \cdot 3(3 \cdot 8-20 \cdot 8)$ & $52 \cdot 6(39 \cdot 7-65 \cdot 6)$ \\
Christchurch, NZ & 26 & $19 \cdot 2(4 \cdot 1-34 \cdot 4)$ & $11 \cdot 5(0-23 \cdot 8)$ & $69 \cdot 2(51 \cdot 5-87 \cdot 0)$ \\
Otago, NZ & 18 & 0 & $5 \cdot 5(0-16 \cdot 1)$ & $94 \cdot 4(83 \cdot 9-100 \cdot 0)$ \\
Medellin, Colombia & 11 & $45 \cdot 5(16 \cdot 0-74 \cdot 9)$ & 0 & $54 \cdot 5(25 \cdot 1-84 \cdot 0)$ \\
Kuala Lumpur, & 12 & $41 \cdot 7(13 \cdot 8-69 \cdot 6)$ & 0 & $58 \cdot 3(30 \cdot 4-86 \cdot 2)$ \\
$\quad$ Malaysia & & & & $54 \cdot 5(25 \cdot 1-84 \cdot 0)$ \\
Suva, Fiji & 11 & $45 \cdot 5(16 \cdot 0-74 \cdot 9)$ & 0 & $48 \cdot 0(41 \cdot 6-54 \cdot 3)$ \\
All regions* & 266 & $41 \cdot 3(34 \cdot 6-47 \cdot 9)$ & $10 \cdot 7(6 \cdot 3-15 \cdot 2)$ & \\
\hline
\end{tabular}

*Calculated giving equal weight to each location (the collection of isolates from various locations in the UK, named 'Various, UK' was treated as one location for the purpose of averaging). 
Table 3. Prevalence of cluster $A$, and of isolates belonging to branches $B$ and $C$, in patients with different characteristics

\begin{tabular}{|lcccc|}
\hline $\begin{array}{l}\text { Patient characteristic or } \\
\text { body location }\end{array}$ & $\begin{array}{c}\text { No. of } \\
\text { isolates/regions* }\end{array}$ & \multicolumn{2}{c|}{ Prevalence in \% $(\mathbf{9 5} \%$ confidence limits $)$} \\
\cline { 3 - 4 } & & Cluster A & Branch B \\
\hline Females & $96 / 9$ & $33 \cdot 4(25 \cdot 8-41 \cdot 0)$ & $9 \cdot 7(4 \cdot 6-14 \cdot 8)$ & $56 \cdot 9(49 \cdot 0-64 \cdot 9)$ \\
Males & $113 / 9$ & $41 \cdot 0(30 \cdot 8-51 \cdot 1)$ & $5 \cdot 9(1 \cdot 9-9 \cdot 8)$ & $52 \cdot 2(42 \cdot 1-62 \cdot 2)$ \\
AIDS & $14 / 5$ & $75 \cdot 6(70 \cdot 1-81 \cdot 0)$ & $2 \cdot 2(0-6 \cdot 3)$ & $22 \cdot 2(18 \cdot 1-26 \cdot 3)$ \\
Neutropenia & $13 / 3$ & $27 \cdot 8(17 \cdot 8-37 \cdot 7)$ & $11 \cdot 1(0-23 \cdot 7)$ & $61 \cdot 1(45 \cdot 1-77 \cdot 1)$ \\
Lower gastrointestinal tract & $22 / 5$ & $45 \cdot 0(40 \cdot 1-49 \cdot 9)$ & $21 \cdot 7(18 \cdot 5-24 \cdot 8)$ & $33 \cdot 3(28 \cdot 0-38 \cdot 7)$ \\
Urine & $26 / 4$ & $54 \cdot 2(40 \cdot 4-68 \cdot 0)$ & $2 \cdot 5(0-7 \cdot 1)$ & $43 \cdot 3(29 \cdot 6-57 \cdot 1)$ \\
Vaginal & $15 / 4$ & $42 \cdot 5(35 \cdot 4-49 \cdot 6)$ & $13 \cdot 3(0-28 \cdot 0)$ & $44 \cdot 2(30 \cdot 0-58 \cdot 3)$ \\
Upper resp. tract (incl. oral sites) & $102 / 9$ & $29 \cdot 0(19 \cdot 1-38 \cdot 9)$ & $12 \cdot 3(5 \cdot 9-18 \cdot 6)$ & $58 \cdot 7(47 \cdot 6-69 \cdot 8)$ \\
Sterile site & $23 / 5$ & $48 \cdot 7(25 \cdot 5-71 \cdot 8)$ & $4 \cdot 0(0-9 \cdot 0)$ & $34 \cdot 0(13 \cdot 8-54 \cdot 2)$ \\
Skin/wounds & $48 / 8$ & $32 \cdot 8(25 \cdot 3-40 \cdot 2)$ & $1 \cdot 3(0-3 \cdot 6)$ & $68 \cdot 1(60 \cdot 6-75 \cdot 5)$ \\
Allf & $472 / 12$ & $43 \cdot 0(31 \cdot 8-54 \cdot 1)$ & $8 \cdot 4(1 \cdot 1-15 \cdot 7)$ & $47 \cdot 4(35 \cdot 4-59 \cdot 4)$ \\
\hline
\end{tabular}

* Number of all isolates from patients having the characteristic and number of regions in which patients with this characteristic were sampled.

† Mean prevalences for a given patient characteristic were calculated giving equal weight to each region in which patients with this characteristic had been sampled.

$\ddagger$ Mean prevalences across all characteristics were calculated giving equal weight to each of the above-listed means of each characteristic; the number of isolates listed exceeds 266 because the same isolate can be associated with several characteristics.

Table 4. Regional specificity of isolates

\begin{tabular}{|c|c|c|c|c|}
\hline $\begin{array}{l}\text { Set of isolates } \\
\text { analysed }\end{array}$ & $\begin{array}{l}\text { Mean genetic } \\
\text { distance within } \\
\text { regions } \pm S D^{*}\end{array}$ & $\begin{array}{l}\text { Mean genetic } \\
\text { distance between } \\
\text { regions } \pm \mathrm{SD}^{*}\end{array}$ & $\begin{array}{l}\text { Mean genetic distance between regions } \\
\text { minus mean genetic distance within } \\
\text { regions (as \% of distance within regions) }\end{array}$ & Significance $\nmid$ \\
\hline All & $0 \cdot 30 \pm 0 \cdot 04$ & $0 \cdot 34 \pm 0 \cdot 04$ & $0 \cdot 04(13 \%)$ & $P<0.0071$ \\
\hline Cluster A & $0 \cdot 16 \pm 0 \cdot 02$ & $0 \cdot 19 \pm 0 \cdot 02$ & $0.03(19 \%)$ & $P<0.0002$ \\
\hline Branch B & $0.32 \pm 0.02$ & $0.33 \pm 0.04$ & $0.01(3 \%)$ & $P<0.8604$ \\
\hline Branch C & $0 \cdot 34 \pm 0.07$ & $0.39 \pm 0.06$ & $0.04(12 \%)$ & $P<0.0465$ \\
\hline
\end{tabular}

*For isolates from Britain obtained from locations other than Leicester and London ('Various, UK'; see Table 1) no distance within region was generated but distances between these isolates and isolates from other regions were generated.

† Unpaired two-tailed $t$-test testing the significance of the difference between genetic distances between isolates from the same geographical region and genetic distances between isolates from different geographical regions. For branch B isolates a paired $t$-test was also carried out $(P<0 \cdot 5214)$.

the distribution of the remaining genetically diverse isolates between branches B and C.

\section{Regional specificity of isolates}

It has been reported that C. albicans isolates from the same region can be genetically more similar to each other than to isolates from other regions (Schmid et al., 1993; Pfaller et al., 1998) but only in a qualitative fashion for a limited number of regions. We therefore used our geographically widespread collection to confirm and quantitatively define this regional specificity and to assess the dependence of genetic distance on geographical distance. We found that in 65 out of 66 possible comparisons between the 12 geographical regions studied, the mean genetic distance between isolates from two different regions was higher than the mean of the genetic distance between isolates within the two regions. Isolates from two different regions were on average $13 \%$ genetically more distant from each other than from isolates originating from the same region (Table 4). When we determined regional specificity for individual branches of the tree shown in Fig. 1 (as the difference between genetic distance between regions and within regions; Table 4) we observed that cluster A showed regional specificity comparable to, or exceeding, that of the genetically much more diverse collection of isolates contained within branches B and C. Cluster $\mathrm{A}$ isolates from different regions were on average $19 \%$ genetically more distant than cluster A isolates from the same region, compared to $3 \%$ for branch $\mathrm{B}$ and $12 \%$ for branch $\mathrm{C}$. The subgroup A1 of cluster A 


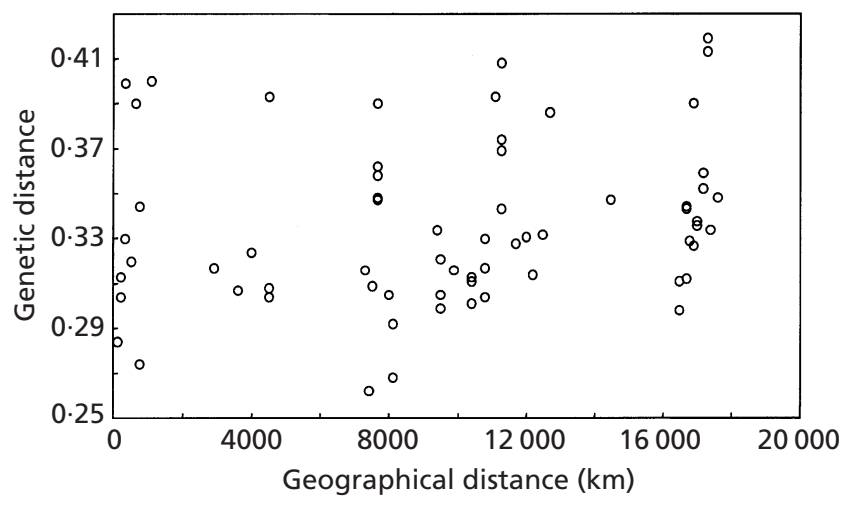

Fig. 4. Relationship between genetic distance and geographical distance between sites of collection of isolates. Mean genetic distances were calculated between each set of isolates from each region listed in Table 1 and sets from all other regions. These were plotted against the respective geographical distances between regions.

showed the highest regional specificity $(46 \%$ higher genetic distance between isolates from different regions than between isolates from the same region).

When we plotted genetic distance between isolates from different regions against geographical distance between the regions (Fig. 4), we found no correlation between genetic distance and geographical distance. Although the mean of the genetic distance between isolates collected $18000 \mathrm{~km}$ apart was slightly $(25 \%)$ larger than the mean distance between isolates from regions less than $1000 \mathrm{~km}$ apart, a linear regression analysis did not support even this weak dependence $\left(R^{2}=0 \cdot 003\right)$.

\section{DISCUSSION}

We have identified a group of highly similar C. albicans isolates, represented by cluster $\mathrm{A}$ in the tree shown in Fig. 1, with a high prevalence across a variety of geographical regions, patient types and types of infections. No other group of highly similar isolates with comparable prevalence was detectable, indicating that this group constitutes the predominant aetiological agent of candidiasis.

It is important to note that very similar mean prevalence values for the group were obtained regardless of whether means were calculated on the basis of the number of isolates in our sample belonging to the cluster, by giving each region the same weight or by giving each patient characteristic the same weight $(37 \%, 41 \%$ and $43 \%$ respectively). This uniformity, in spite of various biases in the collection of isolates analysed, provides additional evidence supporting the ubiquity of the group. It also suggests that the tree depicted in Fig. 1 may at least give an approximation of the relationships between diseasecausing C. albicans isolates in general, especially since the data shown in Fig. 3 suggest the distribution to be fairly stable over time (in most regions; see below). This view is also supported by the observation that the population structure reflected within the tree shown in Fig. 1 resembles that observed in an earlier numerical analysis using a different set of strains and different taxonomic methods (Hunter, 1991). Because of the observed correlations between Ca3-based distances and other markers (Schmid et al., 1995a; Pujol et al., 1997) the tree may also give some indication of the degree of phenotypic diversity and diversity in terms of enzyme locus polymorphisms among infectious isolates.

However, we note that the relationships shown in the tree in Fig. 1 will not always approximate the distribution of isolates in a given patient population. This is exemplified by the high prevalence of a group of branch C strains transmitted in a hospital in Wellington (Schmid et al., 1995b), and a lower prevalence of cluster $A$ in all four New Zealand locales, compared to those observed in the other centres surveyed. It is conceivable, however, that such regional deviations will be reduced over time. Even though there is no statistically significant change in the prevalence of cluster A across all locales with time, it is possible that in some more remote locales such as New Zealand the prevalence of cluster A, whose low genetic diversity indicates that it may be of fairly recent origin, may not yet have reached its maximum value.

The discovery of a group of C. albicans strains more successful than other genotypes in a variety of patient populations and types of infection is not unexpected, given the evidence for C. albicans' clonal mode of reproduction (Odds, 1988; Caugant \& Sandven, 1993; Pujol et al., 1993; Tibayrenc, 1997), supported also by the findings presented here, of highly similar isolates in geographically widely separated regions (Ørskov \& Ørskov, 1983). Clonally reproducing organisms adapt to their environments in two ways. Separate clonal lines may evolve, each of which is adapted to one particular ecological niche, if such niches are stable (frozen niche model). In contrast, clonally reproducing organisms exposed to frequent changes in their environment respond by the evolution of so called general-purpose genotypes, selected on the basis of their adaptability to a variety of niches (Parker et al., 1977; Whittam et al., 1983; Tibayrenc \& Ayala, 1988; Fox et al., 1996; Hermanutz \& Weaver, 1996; Jacobsen \& Forbes, 1997; Semlitsch et al., 1997). In the case of C. albicans the duration for which it can occupy a particular niche on a particular patient is limited by the lifespan of the patient and physiological changes of the patient which may alter that niche. For infection-causing strains a niche will often be extremely short-lived (existing only until the patient is cured or dies). The probability of encountering another patient offering the same type of niche during this time would in most cases be low. Long-term success of a given strain should therefore be enhanced if it is broadly adaptable. In other words one would expect selection to favour a general-purpose genotype, whose existence our data indicate. Given that the incidence of candidosis is much lower than the incidence of commensal colonization (Odds, 1988), such a generalpurpose genotype should also be capable of efficient 
commensal colonization. Our earlier studies support this view, in that the most prevalent groups of diseasecausing strains observed (which we have shown here to be part of the general-purpose cluster A) were also the most prevalent commensal colonizers (Schmid et al., 1990, 1992, 1993, 1995a, b; Hellstein et al., 1993; Schmid, 1993). We note that branches B and C may also contain (considerably less successful) general-purpose genotypes, but the lineages contained in them are too low in prevalence to resolve this issue.

The existence of a general-purpose genotype in $C$. albicans may have bearing on the ongoing debate as to whether C. albicans has a strictly clonal mode of reproduction, simulated by recent phylogenetic studies, which have found evidence of segregation of genetic markers and recombination (Gräser et al., 1996; Tibayrenc, 1997). Other species have been found to forgo sexual recombination when colonizing new niches or variable environments, using clonally reproducing general-purpose genotypes (Parker \& Selander, 1975; Parker et al., 1977; Jacobsen \& Forbes, 1997). If the current major representatives of the species C. albicans have arisen from sexual ancestors fairly recently, phylogenetic analyses would detect remnants of ancestral recombination and segregation even if the modern representatives of the species propagate predominantly or exclusively in a clonal mode.

Our data also provide some indications of how C. albicans spreads between regions. Although we demonstrated regional specificity, we found no significant correlation between the geographical distance between two regions and the genetic distance between isolates obtained from them. This suggests that there is little radial spread of strains between geographically adjacent regions. As far as the general-purpose genotype is concerned, it suggests that once a region has been 'seeded' with members of this group, their progeny will undergo further clonal evolution. Given that the prevalence of lineages represented by branches $\mathrm{B}$ and $\mathrm{C}$ is also fairly uniform across regions, the same may apply to the latter. Our data thus suggest that regional specificity is largely caused by independent clonal evolution within individual regions of sets of ancestral strains common to all regions.

From a clinical perspective the most important implication of our data concerns the selection of C. albicans model strains and the search for pathogenicity factors. The existence of a widespread general-purpose genotype offers the opportunity to pick model strains from a genetically fairly homogeneous group, that outperform other strains as pathogens. We can deduce from the ubiquitous high prevalence of cluster $A$ that its members share traits which allow them to cause disease in a variety of patient types more often than other genotypes, and that this has led to the group's wide distribution. Part of their success as pathogens might stem simply from their high prevalence as commensals. However, we have repeatedly demonstrated that their prevalence as pathogens is significantly higher than their prevalence as commensal colonizers (Schmid et al., 1990, 1992, 1995a, b). Thus they must possess disease-related traits which explain this increase in prevalence. Such traits could include (i) better ability to infect compromised hosts which did not previously carry commensal strains, (ii) the ability to replace existing commensal strains in compromised patients and (iii) increased ability to cause disease once a host (on which they were previously present as commensals) becomes compromised. A comparison of the genomes of cluster A strains with the genomes of other less successful strains thus offers the opportunity to determine these factors (Giblin et al., 1998). It should be noted however that, since cluster A strains constitute a general-purpose genotype, specific virulence traits which benefit C. albicans only when causing disease should be rare since they would constitute a genetic and/or metabolic load when it acts as a commensal and thus be selected against (Dawkins \& Krebs, 1979). More likely virulence traits would be properties which would benefit C. albicans both in situations where it acts as a commensal and when it causes disease, i.e. traits which make it more aggressive as a colonizer and thus more likely to take advantage of breaches in the host's defence.

\section{ACKNOWLEDGEMENTS}

We thank Peter Lockhart, David Penny, Peter Waddell and Mike Hendy for advice regarding the Splitstree analyses, and David Swofford for a pre-release version of PAUP. This work was supported by grants to J.S. from the Massey University Research Foundation and the New Zealand Lottery Grants Board.

\section{REFERENCES}

Caugant, D. A. \& Sandven, P. (1993). Epidemiological analysis of Candida albicans strains by multilocus enzyme electrophoresis. J Clin Microbiol 31, 215-220.

Dawkins, R. \& Krebs, J. R. (1979). Arms races between and within species. In The Evolution of Adaptation by Natural Selection, pp. 55-77. Edited by J. M. Smith \& R. Holliday. Cambridge: Cambridge University Press.

Fox, J. A., Dybdahl, M. F., Jokela, J. \& Lively, C. M. (1996). Genetic structure of coexisting sexual and clonal subpopulations in a freshwater snail (Potamopyrgus antipodarum). Evolution 50, 1541-1548.

Giblin, L., Edelmann, A., Maltzahn, N. B. v., Cleland, S. B. \& Schmid, J. (1998). A DNA fragment associated with the success of Candida albicans strains as pathogens. Abstracts, Sixth International Mycological Congress, Jerusalem, Israel, p. 12.

Gräser, Y., Volovsek, M., Arrington, J., Schönian, G., Presber, W., Mitchell, T. G. \& Vilgalys, R. (1996). Molecular markers reveal that population structure of the human pathogen Candida albicans exhibits both clonality and recombination. Proc Natl Acad Sci USA 93, 12473-12477.

Hellstein, J., Vawter-Hugart, H., Fotos, P., Schmid, J. \& Soll, D. R. (1993). Genetic similarity and phenotypic diversity of commensal and pathogenic strains of Candida albicans isolated from the oral cavity. J Clin Microbiol 31, 3190-3199.

Hermanutz, L. A. \& Weaver, S. E. (1996). Agroecotypes or phenotypic plasticity? Comparison of agrestal and ruderal populations of the weed Solanum ptycanthum. Oecologia 105, 271-280. 
Hunter, P. R. (1991). The sub-specific numerical analysis of Candida albicans. J Med Vet Mycol 29, 105-115.

Jacobsen, R. \& Forbes, V. E. (1997). Clonal variation in life-history traits and feeding rates in the gastropod, Potamopyrgus antipodarum: performance across a salinity gradient. Funct Ecol 11, 260-267.

Lanyon, S. M. (1985). Detecting internal inconsistencies in distance data. Syst Zool 34, 397-403.

Lapointe, F.-J., Kirsch, J. A. W. \& Bleiweiss, R. (1994). Jackknifing of weighted trees: validation of phylogenies reconstructed from distance matrices. Mol Phylogenet Evol 3, 256-267.

Odds, F. C. (1988). Candida and Candidosis, 2nd edn. London: Baillière Tindall.

Ørskov, F. \& Ørskov, I. (1983). Summary of a workshop on the clone concept in the epidemiology, taxonomy, and evolution of the enterobacteriaceae and other bacteria. J Infect Dis 148, 346-357.

Parker, E. D. J. \& Selander, R. K. (1975). The organization of genetic diversity in the parthenogenetic lizard Cnemidophorus tesselatus. Genetics 84, 791-805.

Parker, E. D. J., Selander, R. K., Hudson, R. O. \& Lester, L. J. (1977). Genetic diversity in colonizing parthenogenetic cockroaches. Evolution 31, 836-842.

Pfaller, M. A., Lockhart, S. R., Pujol, C., Swails-Wenger, J. A., Messer, S. A., Edmont, M. B., Jones, R. N., Wenzel, R. P. \& Soll, D. R. (1998). Hospital specificity, region specificity, and fluconazole resistance of Candida albicans bloodstream isolates. J Clin Microbiol 36, 1518-1529.

Pujol, C., Reynes, J., Renaud, F., Raymond, M., Tibayrenc, M., Ayala, F. J., Janbon, F., Mallié, M. \& Bastide, J.-M. (1993). The yeast Candida albicans has a clonal mode of reproduction in a population of infected human immunodeficiency virus-positive patients. Proc Natl Acad Sci USA 90, 9456-9459.

Pujol, C., Joly, S., Lockhart, S. R., Noel, S., Tibayrenc, M. \& Soll, D. R. (1997). Parity among the randomly amplified polymorphic DNA method, multilocus enzyme electrophoresis and Southern blot hybridization with the moderately repetitive DNA probe Ca3 for fingerprinting Candida albicans. J Clin Microbiol 35, 2348-2358.
Schmid, J. (1993). Candidiasis: conclusions from DNA fingerprinting. Clin Adv Treatment Fungal Infect 4, 12-16.

Schmid, J., Voss, E. \& Soll, D. R. (1990). Computer-assisted methods for assessing strain relatedness in Candida albicans by fingerprinting with the moderately repetitive sequence Ca3. J Clin Microbiol 28, 1236-1243.

Schmid, J., Odds, F. C., Wiselka, M. J., Nicholson, K. G. \& Soll, D. R. (1992). Genetic similarity and maintenance of Candida albicans strains from a group of AIDS patients, demonstrated by DNA fingerprinting. J Clin Microbiol 30, 935-941.

Schmid, J., Rotman, M., Reed, B., Pierson, C. L. \& Soll, D. R. (1993). Genetic similarity of Candida albicans strains from vaginitis patients and their partners. J Clin Microbiol 31, 39-46.

Schmid, J., Hunter, P. R., White, G. C., Nand, A. K. \& Cannon, R. D. (1995a). Physiological traits associated with success of Candida albicans strains as commensal colonisers and pathogens. J Clin Microbiol 33, 2920-2926.

Schmid, J., Tay, Y. P., Wan, L., Carr, M., Parr, D. \& McKinney, W. (1995b). Evidence for nosocomial transmission of Candida albicans obtained by $\mathrm{Ca} 3$ fingerprinting. J Clin Microbiol 33, 1223-1230.

Semlitsch, R. D., Hotz, H. \& Guex, G. D. (1997). Competition among tadpoles of coexisting hemiclones of hybridogenetic Rana esculenta: support for the frozen niche variation model. Evolution 51, 1249-1261.

Swofford, D. L., Olsen, G. J., Waddell, P. J. \& Hillis, D. M. (1996). Phylogenetic inference. In Molecular Systematics, pp. 407-514. Edited by D. M. Hillis, C. Moritz \& B. K. Mable. Sunderland, MA: Sinauer Associates.

Tibayrenc, M. (1997). Are Candida albicans natural populations subdivided? Trends Microbiol 5, 253-257.

Tibayrenc, M. \& Ayala, F. J. (1988). Isozyme variability in Trypanosoma cruzi, the agent of Chagas' disease: genetical, taxonomical and epidemiological significance. Evolution 42, 277-292.

Whittam, T. S., Ochman, H. \& Selander, R. K. (1983). Multilocus genetic structure in natural populations of Escherichia coli. Proc Natl Acad Sci 80, 1751-1755.

Received 4 February 1999; revised 25 May 1999; accepted 3 June 1999. 